Philosophie ANTIQUE
Philosophie antique

Problèmes, Renaissances, Usages

$12 \mid 2012$

Autour de la perception

\title{
Suzanne HUSSON, La République de Diogène. Une cité en quête de la nature
}

\section{Louis-André Dorion}

\section{(2)enEdition}

\section{Journals}

Édition électronique

URL : https://journals.openedition.org/philosant/960

DOI : 10.4000/philosant.960

ISSN : 2648-2789

\section{Éditeur}

Éditions Vrin

\section{Édition imprimée}

Date de publication : 1 novembre 2012

Pagination : 307-310

ISBN : 978-2-7574-0400-3

ISSN : $1634-4561$

\section{Référence électronique}

Louis-André Dorion, « Suzanne husson, La République de Diogène. Une cité en quête de la nature », Philosophie antique [En ligne], 12 | 2012, mis en ligne le 01 novembre 2018, consulté le 02 décembre 2022. URL : http://journals.openedition.org/philosant/960 ; DOI : https://doi.org/10.4000/philosant 960

\section{(c) (i) (3)}

Creative Commons - Attribution - Pas d'Utilisation Commerciale - Pas de Modification 4.0 International - CC BY-NC-ND 4.0

https://creativecommons.org/licenses/by-nc-nd/4.0/ 
Platon. Un des points de départ de l'interrogation est un court passage surprenant au début de la conversation - c'est la manière très injuste dont Socrate résume le premier embryon de définition de la piété proposé par Euthyphron (5d8-e3); celui-ci avait essayé de cerner une catégorie des actes pieux, mais Socrate réagit (6d1-3) comme s'il n'avait indiqué qu'un cas singulier ( «la piété, c'est ce que moi je fais »). L'examen des causes possibles de cette manipulation attribuée à Socrate engage Rossetti dans une discussion sur les dialogues aporétiques en général, sur le contexte et la leçon de l'entretien, sur la recherche définitionnelle qu'il considère comme un apport de Platon, ainsi que sur la datation et le cadre narratif. Il m'est impossible de résumer ici ses acquis sans faire tort à la manière très nuancée dont il les présente.

C'est un livre à la fois solide et courageux, écrit par un homme très intelligent.

Agnese GAILE-IRBE.

Suzanne Husson, La République de Diogène. Une cité en quête de la nature, Paris, Librairie philosophique J. Vrin, 2011 (Histoire des doctrines de l'Antiquité classique, 40), 235 p. ISBN 978-2-7116-2265-8.

On compte plusieurs études sur la Politeia attribuée à Diogène, mais, sauf erreur de ma part, aucun livre ne lui avait jusqu'à maintenant été consacré, non plus qu'à la pensée politique de Diogène. L'ouvrage passionnant que Suzanne Husson (désormais S. H.) vient d'y consacrer démontre à l'évidence que la pensée politique de Diogène, et des cyniques en général, méritait une étude approfondie. Dans le premier chapitre de l'ouvrage («Problèmes textuels », p. 21-45), S. H. se penche sur les principaux témoignages relatifs à la Politeia de Diogène, notamment le fameux témoignage de Philodème (Sur les stö̈ciens, P.Herc. 155 et $339=$ SSR V B 126), dont S. H. reproduit le texte (p. 22-23) et fournit une traduction (p. 2325). Après un examen détaillé et rigoureux des arguments pro et contra, elle conclut de façon convaincante à l'authenticité de la Politeia attribuée à Diogène ( $c f$. p. 40) et elle procède ensuite à un examen des parallèles entre la République de Platon et celle de Diogène. Ces parallèles sont suffisamment nombreux et étroits pour justifier la conclusion suivant laquelle la Politeia de Diogène dépend de la République de Platon et y répond en radicalisant les positions de ce dernier ( $c f$. p. 45 ; voir aussi p. 105-132: «Radicaliser Platon »).

L'un des grands mérites de l'ouvrage de S. H., et c'est surtout cet apport que je discuterai, est d'avoir pleinement reconnu l'importance de la recherche de l'autarcie dans le cynisme et d'avoir cherché à comprendre l'articulation entre la recherche de l'autarcie individuelle et le projet collectif d'une cité cynique ( $c f$., entre autres, «La cité de l'autarcie », p. 75-101). Il s'agit d'une question redoutable dont S. H. reconnaît d'entrée de jeu la difficulté ( $c f$. p. 7) : si le sage est autarcique et que la nature lui fournit d'elle-même tout ce dont il a besoin pour réaliser son autarcie, en quoi les cyniques ont-ils besoin d'une cité ? De plus, « n'est-il pas contradictoire dans les termes de vouloir constituer une cité composée d'individus autarciques? »(p.83). Il s'agit d'un vrai problème et un rapide rappel des positions de Platon et d'Aristote permettra de mesurer l'ampleur de la difficulté à laquelle est confrontée S. H. Dans la République, la nécessité pour l'homme de vivre en cité vient de ce que l'homme n'est pas autarcique sur le plan individuel et 
que la satisfaction des besoins vitaux ne peut être assurée que dans le cadre de la cité (II, 369b). Sur ce point, Aristote partage l'avis de Platon et formule une position qui se démarque à peine de celle de son maître : c'est à la cité de réaliser l'autarcie (matérielle) qui est inaccessible à chaque homme pris isolément (Pol. I 2, 1253a2730). Or, étant donné que les cyniques aspirent à l'autarcie individuelle et qu'ils considèrent que la nature leur fournit tout ce qui est nécessaire pour réaliser cette autarcie, pourquoi Diogène, qui est lui-même parvenu à l'autarcie dans l'Athènes de son temps, a-t-il senti le besoin de concevoir une politeia cynique ? Avant d'exposer la réponse que $S$. $H$. donne à cette question, il me paraît important de souligner qu'il n'est pas du tout question de l'autarcie dans le témoignage de Philodème. Certes, ce témoignage est lacunaire, mais étant donné que $S$. H. reconnaît elle-même que « rien ne nous est dit sur la structure générale de l'œuvre ni sur la réflexion politique qui éventuellement s'y faisait jour » (p. 41), on peut légitimement se demander si et dans quelle mesure il était question d'autarcie dans la Politeia de Diogène. En tout état de cause, il faut reconnaître, sans doute plus clairement et plus ouvertement que ne le fait $S$. H., que la question de l'articulation entre la recherche de l'autarcie individuelle et le projet d'une cité cynique n'y était peut-être pas abordée. C'est donc à partir d'autres témoignages sur Diogène que $S$. H. cherche à résoudre ce problème - car il ne fait aucun doute que c'en est un.

$S$. H. résout le paradoxe qui est au cœur de son ouvrage en affirmant que « le cynique, tout autarcique qu'il soit, est néanmoins en quête d'un homme véritable avec qui partager sa vie et sa vertu » (p. $85 ; c f$. aussi p. 86 : « Le sage aura donc besoin d'autrui, quel qu'il soit, pour connaître et progresser dans sa propre vertu. ») La solution du paradoxe initial conduit ainsi à un nouveau paradoxe : le sage qui ne doit son autarcie à personne, si ce n'est à la nature, a néanmoins besoin d'autrui pour « connaître et progresser dans sa propre vertu ». Or cela entraîne que le terme autarkeia ne suppose pas nécessairement une autosuffisance absolue et, de fait, S. H. s'applique à circonscrire les différentes acceptions du terme autarkeia et à identifier les emplois de ce terme qui impliquent une certaine dépendance à l'égard d'autrui (p. 77-78). La position de S. H. ne me convainc pas entièrement pour les raisons suivantes: (1) L'autarcie du sage cynique est plutôt limitée - il a besoin d'autrui pour connaître sa vertu - et elle est bien en deçà de l'autarcie que Socrate reconnaît au sage dans la République (III, 387d-e) et dans le Lysis (215a-b), où il affirme que le sage bon - et donc vertueux - n'a pas besoin d'amis. Or si le sage n'a pas du tout besoin d'amis - comme le soutiennent également d'autres socratiques (cf. SSR II O 33 et IV H 13) -, pas même pour connaître sa vertu et y progresser, il n'a pas non plus besoin de vivre en cité. Si Diogène est bien un héritier de Socrate nous y reviendrons -, voire un «Socrate devenu fou », on peut s'étonner qu'il n'ait pas traité une question qui avait été soulevée par Socrate et d'autres socratiques et qu'il ait attribué au sage cynique une autarcie beaucoup moins radicale que celle reconnue au sage par Socrate. (2) L'affirmation que le sage autarcique a besoin d'autrui pour connaître sa vertu et y progresser ne peut s'autoriser du témoignage de Philodème, et le passage de Plutarque (De prof. in virt. 82) que S. H. cite (p. 86) pour justifier son interprétation me paraît nettement insuffisant pour l'étayer. (3) En outre, la position de S. H. conduit inévitablement à une certaine dévalorisation de l'autarcie, qui me paraît patente dans l'affirmation suivante : « Si 
l'autarcie, en effet, peut être "gage de perfection", nous pouvons également dire qu'elle n'est pas la perfection elle-même, qu'elle n'est pas par elle-même un achèvement $\gg$ (p. 87). Or je ne crois pas que l'autarcie ne soit qu' « une condition négative préalable à la réalisation de l'excellence d'un être » (p. 87-88) et de la vertu. La distinction que S. H. fait entre autarcie et perfection n'est pas corroborée par les textes, qui montrent au contraire que l'autarcie et la perfection sont des synonymes (cf. Philèbe, 67a ; Aristote, EN I 5, 1097b6-8; [Platon], Déf. 412b); en outre, l'autarcie est l'une des principales propriétés de la divinité, et non pas une simple « condition négative » préalable à une forme plus élevée de réalisation, ainsi que le démontrent clairement les textes où le sage qui aspire à la perfection divine cherche à y parvenir par l'imitation de l'autarcie qui caractérise les dieux (cf. Mem. I 6, 10 et D. L. VI 105, cités infra).

La question de l'autarcie, dans le cynisme, est indissociable de la tripartition animal/homme/dieu dans la mesure où l'homme est cet être étrange, voire « dénaturé », qui n'est pas autarcique alors que les animaux et les dieux le sont d'emblée. S.H. explique très bien la nécessité, pour l'homme, d'imiter les animaux afin de se dépouiller de la civilisation qui l'a privé de son autarcie naturelle en multipliant les besoins superflus. La nécessité d'imiter les animaux n'entraîne pas, comme on le soutient souvent, une régression primitiviste ( $c f$. p. 96-101), mais elle pose néanmoins un problème difficile, celui de la hiérarchie entre les hommes, les animaux et les dieux. Si les cyniques proposent le modèle animal afin de parvenir à l'autarcie, faut-il en conclure qu'ils inversent la hiérarchie traditionnelle entre les animaux et les hommes et qu'ils reconnaissent la supériorité des animaux ? S.H. soutient que la tripartition animaux/hommes/dieux n'entraîne aucune hiérarchie (p. 143-144) et que l'autarcie accessible à l'homme n'est pas inférieure à celle des animaux, non plus qu'à celle des dieux, lesquels jouent un rôle purement paradigmatique dans la mesure où les cyniques ne reconnaissaient pas leur existence (p. 160). Je ne suis pas sûr que toutes les formes d'autarcie se valent et que les cyniques ne reconnaissaient pas eux-mêmes la supériorité de l'autarcie divine. Dans une lettre pseudépigraphe attribuée à Cratès, mais qui n'en appartient pas moins à la tradition cynique, on peut en effet lire le passage suivant : « Exercez-vous à avoir peu de besoins (car c'est cela qui nous rapproche le plus de la divinité, et le contraire qui nous en éloigne le plus), et il vous sera permis, dans votre situation qui tient le milieu entre les dieux et les bêtes, de vous rendre semblables à l'espèce supérieure et non à l'espèce inférieure. » ([Cratès], Lettre XI = SSR V H 98 ; trad. Rombi et Deleule.) Ce texte est d'autant plus intéressant qu'il est étroitement lié à deux autres textes (Mem. I 6, 10 et D. L. VI 105, cités infra) qui établissent clairement la supériorité de l'autarcie divine, qui est absolue, sur l'autarcie humaine, qui est forcément incomplète et relative. De plus, l'analyse que fait $S$. H. des différentes acceptions du terme autarkeia semble contredire sa position que toutes les formes d'autarcie sont équivalentes, puisqu'il y a manifestement des formes d'autarcie qui sont plus radicales que d'autres.

Je terminerai par quelques remarques sur la filiation entre Socrate et Diogène. Pour S. H., cette filiation ne fait aucun doute et elle l'affirme à de nombreuses reprises ( $c f$. p. 8,10 n. 2, 12, 42, 62-63, 87, 199). Je regrette qu'elle n'ait pas également considéré des éléments de rupture entre Socrate et Diogène, notamment 
le modèle animal, dont elle considère à raison qu'il est au fondement même du cynisme (p. 71). Or, si l'appel au modèle animal est un acte fondateur du cynisme, et que Socrate n'a par ailleurs jamais prôné l'imitation des animaux, bien au contraire, le cynisme peut-il vraiment être considéré comme une «école » socratique? L'analyse des rapports entre Socrate et Diogène demeure partielle pour autant que S. H. ne tient pas compte des recoupements que l'on peut établir entre Diogène et le Socrate de Xénophon, qui est sans contredit l'un des grands absents de ce livre. Lorsqu'elle parle de Socrate, S. H. a toujours en vue le Socrate de Platon et elle méconnaît ainsi les traits spécifiques du Socrate de Xénophon qui le rapprochent du cynisme. Je donnerai quelques exemples de thèmes à propos desquels il aurait été pertinent de faire intervenir le Socrate de Xénophon. Commençons par l'autarcie. À l'instar de plusieurs spécialistes du cynisme, S. H. fait comme si l'autarcie était un idéal propre au cynisme et qui remonte au cynisme, alors que c'est déjà une caractéristique du Socrate de Xénophon (cf. Mem. I 2, 2 ; I 2, 14 ; I 6, 10 ; IV 7,$1 ;$ IV 8,11 ), et non pas de son (faux) jumeau platonicien. Lorsqu'elle cite un important passage de Diogène Laërce sur Diogène et l'autarcie ( « Diogène disait que s'il appartient aux dieux de n'avoir besoin de rien, il appartient aux gens semblables aux dieux d'avoir besoin de peu de choses », VI 105, cité p. 77), elle semble ignorer que ce passage est lui-même une réminiscence de Mémorables, I 6, 10 : « Pour ma part, je [scil. Socrate] considère que l'absence de besoin est divin et qu'avoir le minimum de besoins est ce qui s'approche le plus du divin; et, comme le divin est parfait, ce qui s'en approche le plus s'approche également de la perfection. » Il en va de même pour la notion de ponos: S. H. fait en effet comme si le ponos était au cynisme ce que l'elenchos et l'intellectualisme étaient à Socrate, alors que le ponos et l'ascèse ne caractérisent pas moins le Socrate de Xénophon (cf. Mem. I 2, 1-2 ; II 1, 3 ; II 1, 15 ; II 1, 18-20, etc.) que le cynisme. À la page 88, S. H. affirme avec justesse que l'ascèse du cynique, « dont le but est l'autarcie, ne débouche pas seulement sur une libération intérieure mais libère également le rapport à autrui »; autrement dit, l'autosuffisance n'est pas un obstacle aux liens de philia, contrairement à ce que soutient le Socrate de Platon dans le Lysis (214d-215c); or le Socrate de Xénophon affirme précisément que l'autarcie, loin d'être un empêchement à l'amitié, en est au contraire une condition (cf. Mem. II 6, 1). Enfin, non seulement S. H. méconnaît la parenté entre le Socrate de Xénophon et les cyniques sur certains points essentiels, mais elle le dépossède de certaines doctrines qui lui appartiennent, notamment son développement sur les lois non écrites (Mem. IV 4), que, dans la foulée d'Untersteiner, elle a tort d'attribuer à Hippias (cf.p. 153).

Je serais consterné si les critiques et les objections que j'ai formulées donnaient au lecteur de ce compte rendu une impression négative à l'endroit du livre de S. H. Je me suis attaché à certains aspects qui me paraissent plus problématiques, mais ces réserves n'entament en rien l'admiration et le jugement extrêmement favorable que m'inspire cet ouvrage qui saura s'imposer comme une étude de référence sur la Politeia de Diogène et sur la pensée politique des cyniques.

Louis-André DORION. 